\title{
Bequem, aber fehleranfällig: Die Dunkelziffer ungewerteter Briefwahlstimmen
}

\author{
Dominic Nyhuis
}

Eingegangen: 28. September 2020 / Überarbeitet: 7. August 2021 / Angenommen: 6. Oktober 2021 /

Online publiziert: 5. November 2021

(C) Deutsche Vereinigung für Politikwissenschaft 2021

Zusammenfassung Seit Jahren erfreut sich die Briefwahl steigender Beliebtheit. Während Wählerinnen und Wähler die Bequemlichkeit der Briefwahl schätzen, bleibt in der öffentlichen Debatte häufig unbeachtet, wie anfällig Wahlbriefe für Formfehler sind. Aus vielerlei Gründen werden eingegangene Wahlbriefe von den Wahlleitungen zurückgewiesen. Da diese Stimmen in Deutschland als nicht abgegeben gewertet werden, unterschätzt der in der Wahlstatistik berichtete Anteil ungültiger Briefwahlstimmen das Phänomen nicht gewerteter Briefwahlstimmen ganz erheblich. Der in dieser Arbeit geschätzte Anteil ungewerteter Briefwahlstimmen liegt zwischen 3,2 und 4,0\% und somit deutlich über dem typischen Anteil ungültiger Urnenwahlstimmen. Die Arbeit schließt mit der Forderung, die Zahl der zurückgewiesenen Wahlbriefe künftig in der Wahlstatistik auszuweisen, um Wählerinnen und Wählern eine reflektierte Entscheidung über das Risiko einer ungewerteten Briefwahlstimme zu ermöglichen.

Schlüsselwörter Wahlgesetze · Briefwahl · Ungültige Stimmen · Offizielle Wahldaten · Wahlstatistik

Stand: Oktober 2021

Dominic Nyhuis $(\bowtie)$

University of North Carolina at Chapel Hill, Chapel Hill, USA

E-Mail: nyhuis@unc.edu 


\title{
Convenient, but Prone to Error: Invisible Uncounted Mail Ballots
}

\begin{abstract}
Voting by mail is increasingly popular. Although voters enjoy the convenience of voting by mail, the public is unaware how prone to error mail ballots are. Election administrations reject mail ballots for a variety of reasons. Because German electoral laws treat rejected ballots as not having been cast, the reported number of invalid mail ballots underestimates the true share of uncounted mail ballots. Between $3.2 \%$ and $4.0 \%$ of the mailed ballots in German elections are estimated to remain uncounted, which is far higher than the share of invalid in-person votes. This paper argues that rejected mail ballots should be reported in the official records to make voters aware of the risks of an uncounted mail ballot.
\end{abstract}

Keywords Electoral laws - Voting by mail - Invalid votes - Official voting records · Election statistics

\section{Einleitung: Ungesehene Formfehler bei der Briefwahl}

Seit Jahren erfreut sich die Briefwahl in Deutschland wachsender Beliebtheit. Bei der Bundestagswahl 2017 etwa wurde mehr als jede vierte Stimme per Briefwahl abgegeben, bei der Bundestagswahl 2021 gar 47,3\%. Eine besondere Dringlichkeit haben Debatten um die Briefwahl im Zuge der Covid-19-Pandemie erhalten, als Möglichkeiten gefunden werden mussten, um demokratische Erfordernisse mit der Gesundheit von Wählerinnen und Wählern in Einklang zu bringen (Orlowski und Pohlmann 2020). So wurden die Stichwahlen der bayerischen Kommunalwahlen im März 2020 beispielsweise vollständig per Briefwahl durchgeführt (Pollex et al. 2021). Aber auch schon vor diesem Ausnahmezustand haben sich immer mehr Wählerinnen und Wähler von der Bequemlichkeit der Briefwahl überzeugen lassen. Gesetzlich wurde dem Wunsch nach einer bequemeren Stimmabgabe durch die Einführung der begründungslosen Briefwahl Rechnung getragen, die den Briefwähleranteil noch zusätzlich befördert hat.

Während Wählerinnen und Wähler die Bequemlichkeit der Briefwahl schätzen, gibt es durchaus kritische Stimmen an dieser Entwicklung. Besonders im Vorfeld der US-Präsidentschaftswahlen 2020 hat die Briefwahl eine erhebliche politische Sprengkraft entfaltet. Vordergründig ging es dabei um vermeintlichen Wahlbetrug im Zuge der möglichen Ausweitung der Briefwahl. Tatsächlich lag der Diskussion aber eher die Frage zugrunde, ob und wie die Briefwahl das Wahlergebnis beeinflussen könnte, wenn politikfernen Schichten die Wahrnehmung ihres Wahlrechts erleichtert wird.

In der deutschen Debatte werden vornehmlich konzeptionelle Einwände gegen die Briefwahl vorgetragen, sei es der ungleiche Informationsstand der Wählerinnen und Wähler während des vorgezogenen Briefwahlaktes oder das nicht zu gewährleistende Wahlgeheimnis (Buchstein 2000; Kersting 2019). Die praktischen Aspekte der Briefwahl wurden dagegen bisher selten problematisiert. Die vorliegende Arbeit weist auf die Anfälligkeit der Briefwahl für Formfehler hin. Aufgrund der Zurückweisung von Wahlbriefen durch die Wahlleitungen liegt der Anteil ungewerteter 
Briefwahlstimmen deutlich über dem Anteil ungültiger Urnenwahlstimmen und klar über dem in der Wahlstatistik berichteten Anteil ungültiger Briefwahlstimmen.

Die Differenz zwischen den ungewerteten und den ungültigen Briefwahlstimmen entsteht durch eine missliche Regelung in der deutschen Wahlgesetzgebung. Aufgrund von Formfehlern zurückgewiesene Wahlbriefe werden als nicht abgegeben gewertet, sodass sie in der Wahlstatistik ausschließlich in der nicht realisierten Wahlbeteiligung auftauchen. Praktisch gehen somit zahlreiche Stimmen ungesehen verloren. Die fehlende Berücksichtigung der zurückgewiesenen Wahlbriefe in der Wahlstatistik führt zu einem mangelnden Problembewusstsein, da der berichtete Anteil ungültiger Briefwahlstimmen fast immer unterhalb des Anteils ungültiger Urnenwahlstimmen liegt.

Das fehlende Bewusstsein für die Problematik der ungewerteten Briefwahlstimmen lässt sich selbst in den Veröffentlichungen des Bundeswahlleiters nachweisen. Dort heißt es beispielsweise nach der Bundestagswahl 2017:

Ein möglicher Grund für die niedrigen Anteile ungültiger Stimmen bei den Briefwählerinnen und -wählern könnte sein, dass sie ihren Stimmzettel in Ruhe in ihrer vertrauten Umgebung ausfüllen können und genügend Zeit für das Durchlesen der Hinweise besitzen. Außerdem könnte vermutet werden, dass die Wahlberechtigten, die ihre Stimme bewusst ungültig abgeben wollen, womöglich gar nicht erst aktiv werden und Briefwahlunterlagen beantragen. (Bundeswahlleiter 2018, S. 27)

Der Bundeswahlleiter ignoriert hier, dass Wählerinnen und Wähler mehr Fehler bei der Briefwahl als bei der Urnenwahl machen können und dass deshalb zahlreiche Stimmen verloren gehen, bevor es überhaupt zur Auszählung kommt.

Die vorliegende Arbeit soll dazu beitragen, das Bewusstsein für die Problematik ungewerteter Briefwahlstimmen zu erhöhen. Die konkreten Ziele dieses Beitrags lassen sich in drei Punkten zusammenfassen. Erstens wird das Phänomen der ungewerteten Briefwahlstimmen erläutert. Zweitens werden das Ausmaß der ungewerteten Briefwahlstimmen und die Gründe für die Zurückweisung von Wahlbriefen untersucht. Drittens werden praktische Handlungsempfehlungen formuliert. Künftig sollten Daten über zurückgewiesene Wahlbriefe gesammelt und veröffentlicht werden. Nur so können sich die Wählerinnen und Wähler eine begründete Meinung über die Gefahr einer ungewerteten Briefwahlstimme bilden. Zudem kann die Debatte über die Zurückweisung von Wahlbriefen auf eine bessere empirische Basis gestellt werden, um weitere Reformpotenziale zu identifizieren.

Zur Untersuchung der ungewerteten Briefwahlstimmen wird das folgende Vorgehen gewählt. Nach einem Überblick über die bestehende Forschung werden die zentralen Regelungen der deutschen Wahlgesetze diskutiert. Im nächsten Schritt wird die Erfassung ungewerteter Wahlbriefe diskutiert, die in der vorliegenden Arbeit auf Grundlage von Daten ausgewählter kreisfreier Städte geschieht. Auf dieser Basis kann ein erster Eindruck des Phänomens ungewerteter Briefwahlstimmen gewonnen werden. Da die zurückgewiesenen Wahlbriefe in der Wahlstatistik bisher nicht veröffentlicht werden, ist einschränkend zu bemerken, dass es sich nicht um eine repräsentative Stichprobe handelt und die Daten somit nur einen Orientierungspunkt bieten können. 
Die Daten werden auf zwei Ebenen ausgewertet. Zum einen wird das Aufkommen ungewerteter Wahlbriefe untersucht, das in der vorliegenden Arbeit zwischen 3,2 und 4,0\% aller eingegangenen Wahlbriefe (95\%-Konfidenzintervall) und somit klar über dem mittleren Anteil ungültiger Urnenwahlstimmen liegt. Zum anderen werden die Gründe für die Zurückweisung von Wahlbriefen betrachtet, die vor allem auf Probleme mit dem Wahlschein zurückzuführen sind.

\section{Bestehende Forschung zur Briefwahl und zur ungültigen Stimmabgabe}

Im Zuge der wachsenden Beliebtheit der Briefwahl hat sich die politikwissenschaftliche Forschung verstärkt mit diesem wichtigen Element demokratischer Teilhabe beschäftigt (Gronke et al. 2008). Während es jedoch gerade in der internationalen Forschung eine Vielzahl von Beiträgen zu neueren Formen der Wahlteilnahme gibt, so lassen sich doch erhebliche Lücken in der Literatur feststellen. Der Großteil der bestehenden Forschung stellt die Frage nach dem Effekt der Briefwahl auf die Wahlbeteiligung (Kousser und Mullin 2007; Gerber et al. 2013; Karp und Banducci 2000, 2001; Richey 2008; Southwell 2004, 2009; Gronke und Miller 2012), wobei die Forschung hier mehrheitlich einen positiven Effekt der Briefwahl auf die Beteiligungsneigung konstatiert. Mittelbar steht damit auch der Effekt der Briefwahl auf das Wahlergebnis im Fokus (Thompson et al. 2020; Hanmer und Traugott 2004; Southwell und J. Burchett 2000), der in der Forschung eher verneint wird. Weitere Arbeiten haben sich mit den soziodemografischen Merkmalen der Briefwählerinnen und -wähler beschäftigt (Berinsky et al. 2001; Barreto et al. 2006; Wagner und Lichteblau Im Erscheinen), die sich in der Tendenz aus älteren Wählerinnen und Wählern aus höheren sozialen Schichten zusammensetzen.

Fast unbeachtet sind bisher dagegen praktische Aspekte der Stimmabgabe per Briefwahl geblieben. Alvarez et al. (2013) beschäftigen sich mit dem Zusammenhang zwischen der Briefwahl und nicht abgegebenen Stimmen bei Wahlen im USBundesstaat Kalifornien. Die Autoren zeigen, dass die Briefwahl den Anteil nicht abgegebener Stimmen bei gleichzeitig durchgeführten Wahlen erhöht. Vergleichbar mit dem Interesse der vorliegenden Arbeit beschäftigen sich Alvarez et al. (2008) mit dem Anteil zurückgewiesener Wahlbriefe bei einer Wahl in Los Angeles. Der Beitrag kommt zu dem Schluss, dass 3,9\% der nicht im Wahllokal abgegebenen Stimmen von den Wahlleitungen zurückgewiesen wurden. Darüber hinaus können Zusammenhänge zwischen verschiedenen Strukturmerkmalen und der Wahrscheinlichkeit zurückgewiesener Wahlbriefe festgestellt werden, etwa höhere Zurückweisungsraten für Wählerinnen und Wähler im Ausland, Jungwählerinnen und -wähler sowie Wählerinnen und Wähler, die spanischsprachige Wahlunterlagen nutzen.

An dieser Stelle besteht eine enge Verwandtschaft $\mathrm{zu}$ einem weiteren Literaturstrang, der sich mit den Determinanten der ungültigen Stimmabgabe beschäftigt. Während diese Forschung allerdings wichtige konzeptionelle Anknüpfungspunkte für die vorliegende Arbeit bietet, so stehen in diesem Forschungszweig ungültige Stimmzettel im Vordergrund, während ungültige Briefwahlstimmen bisher nicht gezielt in den Blick genommen wurden. 
Ein Kernelement der Forschung zu ungültigen Stimmen ist die Frage, inwiefern ungültige Stimmen auf bewusste Entscheidungen oder auf Irrtümer von Wählerinnen und Wählern zurückgeführt werden können (Lavies 1968; Solvak und Vassil 2015; Uggla 2008; McAllister und Makkai 1993; Power und Garand 2007; Kouba und Lysek 2019; Moral 2016; Driscoll und Nelson 2014). Dabei konstatiert die Forschung oftmals nicht unerhebliche Anteile bewusst ungültiger Stimmen, die auch bereits für den deutschen Fall identifiziert werden konnten (Fatke und Heinsohn 2017; Baasen 2005; Lavies 1968). Eng verknüpft mit dieser Frage ist die soziodemografische Zusammensetzung der ungültig stimmenden Wählerschaft (Knack und Kropf 2003; Sinclair und Alvarez 2004; Tomz und van Houweling 2003; Herron und Sekhon 2003; Buchler et al. 2004). In der vornehmlich US-amerikanischen Literatur wurde häufig beobachtet, dass ethnische Minderheiten systematisch häufiger ungültige Stimmen abgeben, was allerdings sowohl auf bewusste Protestwahlstimmen als auch auf Bildungsfaktoren zurückgeführt werden könnte.

Speziell mit Blick auf die Briefwahl gibt es neben der groben Unterteilung zwischen bewusst und unbewusst ungültigen Stimmzetteln noch eine weitere denkbare Unterform der bewusst ungültigen Stimmabgabe, nämlich eine ungültige Stimme aufgrund versuchten Wahlbetrugs. Wie im folgenden Abschnitt dargelegt wird, ist die vergleichsweise hohe Komplexität der Briefwahl auf Maßnahmen zur Verhinderung von Wahlbetrug und zur Gewährleistung des Wahlgeheimnisses zurückzuführen. Derartige Maßnahmen erschweren die Stimmabgabe und erhöhen die Gelegenheit für Formfehler und somit die Wahrscheinlichkeit unbewusst ungültiger Stimmabgaben.

Der Zusammenhang zwischen der Komplexität des Wahlaktes und der Wahrscheinlichkeit ungültiger Stimmen ist in der politikwissenschaftlichen Forschung mittlerweile fest etabliert. So gibt es beispielsweise in den USA eine umfangreiche Forschung zu den Effekten verschiedener Wahltechnologien auf ungültige Stimmen (Hanmer et al. 2010; Warf 2006; Ansolabehere und Stewart 2005; Kimball und Kropf 2008; Zucco und Nicolau 2016). Aber selbst bei klassischen Papierstimmzetteln lassen sich problematische Effekte der Stimmzettelgestaltung auf die Wahrscheinlichkeit fehlerhafter Stimmabgaben nachweisen (Herrnson et al. 2012; Wand et al. 2001; Carman et al. 2008; Kimball und Kropf 2005; Pachón et al. 2017). So untersuchen beispielsweise Wand et al. (2001) das Abstimmungsverhalten mit dem bekannten „Butterfly“-Stimmzettel, der bei der US-Präsidentschaftswahl 2000 in Florida zur Anwendung kam. Die Autoren kommen zu dem Schluss, dass die unglückliche Anordnung der Kandidatennamen auf dem Stimmzettel zur Folge hatte, dass zahlreiche Stimmen für den Kandidaten der demokratischen Partei ungewollt für den Kandidaten einer dritten Partei abgegeben wurden. Carmen et al. (2008) zeigen auf Grundlage eines natürlichen Experiments bei der schottischen Parlamentswahl 2007, dass unklare Nutzungshinweise auf dem Stimmzettel zu vielen nicht abgegebenen Stimmen führten. Dieser Befund wird durch die Arbeit Kimball und Kropf (2005) gestützt. Die Autoren kodieren die Gestaltungsmerkmale einer Stichprobe von Stimmzetteln, die bei US-Wahlen zur Anwendung kamen. Kimball und Kropf können zeigen, dass schlechte Designs mit einem systematisch höheren Aufkommen fehlerhafter Stimmzettel einhergehen. 
Das Gebot eines möglichst leicht zu gestaltenden Wahlaktes lässt sich nicht zuletzt dadurch begründen, dass die Ausgestaltung der Wahl alle Wählerinnen und Wähler betrifft. Hierdurch ergeben sich besondere Herausforderungen für die Wahlverwaltungen, da selbst kleinere Hürden in der Praxis den Ausschluss von mehreren Hundert Wählerstimmen bedeuten können. Dabei ist Komplexität selbstverständlich nur im Vergleich der beiden Wahlmodi zu verstehen, wobei die Stimmabgabe per Briefwahl an mehr Stellen misslingen kann als die Urnenwahl.

Weiterhin schließt der Fokus auf ungewollte Formfehler bei der Briefwahl nicht aus, dass auch bei der Briefwahl bewusst ungültige Stimmen oder Fälle von versuchtem Wahlbetrug auftreten. Unabhängig von den Anteilen der bewusst oder unbewusst ungültig stimmenden Wählerinnen und Wählern geht es diesem Beitrag vor allem um die zusätzlichen Möglichkeiten ungewollter Formfehler bei der Briefwahl. Selbst wenn nur eine Teilmenge der Briefwahlstimmen aufgrund ungewollter Formfehler verloren geht, so ist es das Anliegen dieser Arbeit, auf diese verlorenen Stimmen aufmerksam zu machen, die aufgrund der Wahlpraxis unsichtbar bleiben.

Die bestehende Forschung kann in zwei Punkten zusammengefasst werden. Erstens wissen wir grundsätzlich wenig über die Praxis der Briefwahl und wie häufig und an welchen Stellen die Briefwahl misslingt - weder auf der internationalen Ebene noch im Kontext deutscher Wahlen. Diese Lücke in der bestehenden Forschung ist besonders problematisch, da die Briefwahl im Regelfall komplexer ist als die Urnenwahl und somit zusätzliche Potenziale für fehlerhafte Stimmabgaben bestehen. Vor dem Hintergrund des bekannten Zusammenhangs zwischen der Komplexität des Wahlakts und der Wahrscheinlichkeit ungültiger Stimmen, scheint es angezeigt, einen empirischen Blick auf das Ausmaß ungewerteter Briefwahlstimmen und deren Gründe zu erhalten. Zu diesem Zweck werden in diesem Beitrag ausgewählte deutsche Wahlen untersucht. Zur Rahmung der Analyse wird dazu im folgenden Abschnitt zunächst das deutsche Briefwahlverfahren erläutert.

\section{Die Grundlagen der Briefwahl in Deutschland}

Zur Darstellung des deutschen Briefwahlverfahrens werden das Bundeswahlgesetz und die Bundeswahlordnung in den Blick genommen. Da die Landes- und Kommunalwahlgesetze in der Ausgestaltung der Briefwahl eng an das Bundeswahlgesetz angelehnt sind, gelten die Darstellungen vergleichbar für Wahlen auf der Landesund Kommunalebene.

Der erste Schritt zur Briefwahl ist im Regelfall die Versendung der Wahlbenachrichtigung, die umseitig einen Antrag auf Erteilung eines Wahlscheins enthält. Nach Rücksendung des ausgefüllten Antrags werden den Wählerinnen und Wählern die Briefwahlunterlagen zugesendet, die üblicherweise aus fünf Bestandteilen bestehen, die sich in der konkreten Ausgestaltung aber zwischen den Bundesländern unterscheiden. Dies sind der Wahlschein, der insbesondere die eidesstattliche Erklärung enthält, die dem Wahlbrief unterschrieben beizulegen ist. Weiterhin enthalten die Briefwahlunterlagen den Stimmzettel, den Stimmzettelumschlag, den Wahlbriefumschlag und ein Merkblatt. Um eine gültige Briefwahlstimme abzugeben, wird der Stimmzettel wie bei der Urnenwahl ausgefüllt und in den Stimmzettelumschlag 

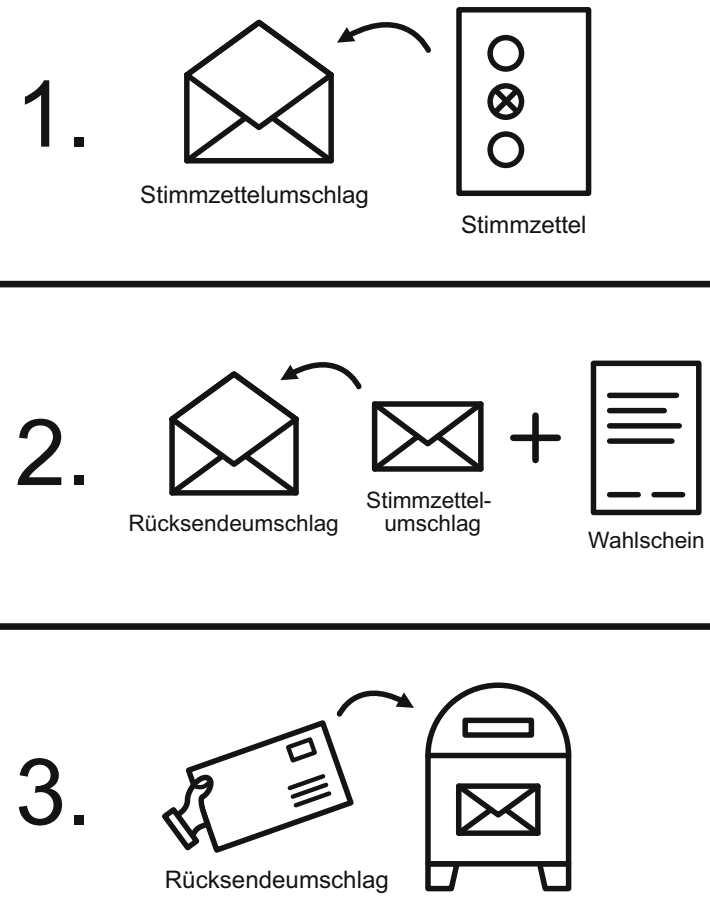

Abb. 1 Die Briefwahl in Deutschland

gesteckt. Der verschlossene Stimmzettelumschlag wird gemeinsam mit dem unterschriebenen Wahlschein in den Wahlbriefumschlag gesteckt, der ebenfalls verschlossen und an das Wahlamt zurückgesendet wird. Das Briefwahlverfahren ist in Abb. 1 skizziert. ${ }^{1}$

Das ohnehin schon aufwendige Briefwahlverfahren wird noch komplexer, wenn mehrere Wahlen parallel durchgeführt werden und die Wählerinnen und Wähler noch mehr Stimmzettel und mitunter Umschläge zu organisieren haben. ${ }^{2}$ Wie in der Analyse gezeigt wird, liegt der Anteil ungewerteter Briefwahlstimmen bei parallel durchgeführten Wahlen noch einmal oberhalb des ohnehin schon erhöhten Anteils ungewerteter Briefwahlstimmen im Vergleich zur ungültigen Urnenwahl. Während

\footnotetext{
${ }^{1}$ Ein besonderer Dank gilt Felix Münchow für die Erstellung der Grafiken zum deutschen Briefwahlverfahren.

2 Als eklatantes Beispiel für die Tücken der parallelen Briefwahl sei auf den Volksentscheid über den Weiterbetrieb des Flughafens Tegel verwiesen, der parallel mit der Bundestagswahl 2017 in Berlin durchgeführt wurde. Die Wählerinnen und Wähler erhielten in diesem Fall einen Stimmzettel für die Bundestagswahl und einen Stimmzettel für den Volksentscheid, aber nur einen Stimmzettelumschlag und einen Rücksendeumschlag. Rund 100.000 Wählerinnen und Wähler steckten nur den Stimmzettel für die Bundestagswahl in den Stimmzettelumschlag, den Stimmzettel für den Volksentscheid dagegen zusammen mit dem Wahlschein in den Rücksendeumschlag. Entsprechend der gesetzlichen Vorgaben (siehe Fußnote 4) wurden diese Stimmen aufgrund der denkbaren Verletzung des Wahlgeheimnisses nicht zur Wahl zugelassen (https://www.rbb24.de/politik/wahl/tegel/beitraege/volksentscheid-briefwaehler-stimmen-verloren. html).
} 
die Zusammenlegung von Wahlen also neben dem Argument der eingesparten Kosten häufig mit der erhöhten Wahlbeteiligung bei nachrangigen Wahlen begründet wird (Vetter 2015), geht die Zusammenlegung von Wahlen doch mit unsichtbaren Beteiligungsverlusten einher, da sich durch die Zusammenlegung von Wahlen der Anteil der ungewerteten Briefwahlstimmen weiter erhöht. Zumindest für die nachrangigen Wahlen gilt dabei allerdings, dass der Beteiligungszugewinn die Verluste durch ungewertete Briefwahlstimmen klar übersteigt.

Die deutschen Wahlgesetze kennen nicht weniger als acht Gründe für die $\mathrm{Zu}$ rückweisung von Wahlbriefen. ${ }^{3}$ Die Dunkelziffer ungewerteter Briefwahlstimmen entsteht, da zurückgewiesene Wahlbriefe keine ungültigen Stimmen sind. In den Worten des Wahlgesetzes: „Die Einsender zurückgewiesener Wahlbriefe werden nicht als Wähler gezählt; ihre Stimmen gelten als nicht abgegeben“ ( $\$ 39$ Abs. 4 Bundeswahlgesetz). An dieser Stelle wurde eine unglückliche Regelung gewählt, da auf diese Weise eine Vielzahl faktisch ungültiger Stimmen unsichtbar bleiben. Anders formuliert können Wählerinnen und Wähler eine ungültige Briefwahlstimme nur dann abgeben, wenn ihre Wahlbriefe zuvor von den Wahlleitungen angenommen wurden.

Grundlegend für die Wertung der zurückgewiesenen Wahlbriefe als Nichtwähler sind Überlegungen zu möglichem Wahlbetrug und daraus resultierende Ungenauigkeiten in der Wahlstatistik. Da Wählerinnen und Wähler mit einem Wahlschein ihre Stimmen auch am Wahltag im Wahllokal abgeben können, werden Wahlbriefe ohne beigelegten Wahlschein von den Wahlleitungen zurückgewiesen (Grund 2). Auf diese Weise soll ausgeschlossen werden, dass Wählerinnen und Wähler sich mehrfach an der Wahl beteiligen. Die entsprechenden Wahlbriefe werden nicht als ungültige Stimmen gewertet, da sie anderenfalls mehrfach in die Zahl der teilnehmenden Wählerinnen und Wähler eingehen und somit die Wahlbeteiligung überschätzen würden.

Vergleichbar lassen sich die Zurückweisungsgründe 5 und 6 motivieren. Wenn dem Wahlbriefumschlag mehrere Stimmzettelumschläge, aber nicht ausreichend Wahlscheine beiliegen (Grund 5), dann kann nicht ausgeschlossen werden, dass einzelne Stimmen doppelt abgegeben wurden. Auch die fehlende Unterschrift auf

\footnotetext{
3 Laut Wahlgesetz sind Wahlbriefe zurückzuweisen, wenn ,1. Der Wahlbrief nicht rechtzeitig eingegangen ist, 2. dem Wahlbriefumschlag kein oder kein gültiger Wahlschein beiliegt, 3. dem Wahlbriefumschlag kein Stimmzettelumschlag beigefügt ist, 4. weder der Wahlbriefumschlag noch der Stimmzettelumschlag verschlossen ist, 5. der Wahlbriefumschlag mehrere Stimmzettelumschläge, aber nicht eine gleiche Anzahl gültiger und mit der vorgeschriebenen Versicherung an Eides Statt versehener Wahlscheine enthält, 6. der Wähler oder die Hilfsperson die vorgeschriebene Versicherung an Eides Statt zur Briefwahl auf dem Wahlschein nicht unterschrieben hat, 7. kein amtlicher Stimmzettelumschlag benutzt worden ist, 8. ein Stimmzettelumschlag benutzt worden ist, der offensichtlich in einer das Wahlgeheimnis gefährdenden Weise von den übrigen abweicht oder einen deutlich fühlbaren Gegenstand enthält." ( 339 Abs. 4 Bundeswahlgesetz). Die Zurückweisungsgründe in den Landes- und Kommunalwahlgesetzen und -ordnungen orientieren sich eng am Bundeswahlgesetz. Manche Wahlgesetze weichen leicht in den spezifischen Formulierungen vom Bundeswahlgesetz ab. Nur wenige Wahlgesetze weichen auch in der Zahl der genannten Gründe ab. In der Regel sind die materiellen Zurückweisungsgründe aber identisch zum Bundeswahlgesetz. So sind die Zurückweisungsgründe bei abweichender Zahl materiell identisch zum Bundeswahlgesetz im bayerischen Landtags- und im niedersächsischen Kommunalwahlgesetz mit je sieben Gründen sowie im Landtags- und Kommunalwahlgesetz des Landes Mecklenburg-Vorpommern, das als einziges Wahlgesetz die Annahme von Wahlbriefen in vier Punkten positiv formuliert. Leicht materiell unterschiedlich ist das Landtagswahlgesetz in Hamburg mit sieben Gründen und das bayerische Kommunalwahlgesetz mit zehn Gründen.
} 
dem Wahlschein (Grund 6) kann mit einem denkbaren Wahlbetrug begründet werden. Im unwahrscheinlichen Fall, dass mehrere Wahlscheine für denselben Wähler eingehen, dann kann die Unterschrift auf dem Wahlschein zur Entscheidung über den gültigen Wahlbrief beitragen. Umgekehrt und naheliegender soll die Unterschrift unter der eidesstattlichen Erklärung einen möglichen Wahlbetrug schon im Vorfeld unterbinden.

Die weiteren Gründe für die Zurückweisung von Wahlbriefen können grob drei Kategorien zugeordnet werden, die sich jedoch weniger offensichtlich aus der Sorge um Wahlbetrug ergeben. Ein fehlender Stimmzettel (Grund 3) schließt die Stimmenzählung offensichtlich aus. Nach Schließung der Wahllokale eingegangene Wahlbriefe werden von der Zählung ausgeschlossen (Grund 1), damit Stimmen nicht strategisch nach Veröffentlichung des Wahlergebnisses abgeben werden können. Die weiteren Gründe ergeben sich aus Bedenken um die Wahrung des Wahlgeheimnisses, nämlich nichtverschlossene Umschläge (Grund 4), ein nichtamtlicher Stimmzettelumschlag (Grund 7) sowie andere Abweichungen des Stimmzettelumschlags (Grund 8).

Während die Zurückweisungsgründe also durchaus berechtigt sind, so ist die zentrale Forderung dieser Arbeit, das Aufkommen zurückgewiesener Wahlbriefe künftig gesondert in der Wahlstatistik auszuweisen. Gerade weil ungewollte Formfehler nicht unwahrscheinlich sind, eigenen sich die Zurückweisungsgründe nicht, um die Öffentlichkeit über die Häufigkeit zurückgewiesener Wahlbriefe im Dunkeln zu lassen. Knapp formuliert wurde mit dem Verschweigen der zurückgewiesenen Wahlbriefe die schlechteste der drei naheliegenden Möglichkeiten gewählt. Besser wäre die Einbeziehung der zurückgewiesenen Wahlbriefe in die ungültigen Stimmen trotz denkbarer Ungenauigkeiten in der Zahl der Wählerinnen und Wähler. Noch besser wären die gesonderte Ausweisung von zurückgewiesenen Wahlbriefen und deren Gründe in der Wahlstatistik.

\section{Die Erfassung ungewerteter Wahlbriefe}

Die Zahl der zurückgewiesenen Briefwahlstimmen ist in der Regel nicht nachvollziehbar. Da die zurückgewiesenen Wahlbriefe als nicht abgegeben gewertet werden, tauchen sie in der Wahlstatistik nicht auf und sind dort schlicht Teil der nicht realisierten Wahlbeteiligung. Während die Zahlen jedoch nicht veröffentlicht werden, haben die Wahlleitungen durchaus Zugang zu Informationen über das Aufkommen der zurückgewiesenen Wahlbriefe. So werden in den Wahlbezirken Niederschriften angefertigt, die unter anderem die Zahl der zurückgewiesenen Wahlbriefe und die Zurückweisungsgründe enthalten. Die hier anfallenden Daten werden im Regelfall jedoch nicht zentral zusammengetragen.

Um einen Eindruck der Häufigkeit von Wahlbriefzurückweisungen zu erhalten, wurden Anfragen an die kommunalen Wahl- und Statistikämter der kreisfreien Städte mit der Bitte um Zusammenstellung der entsprechenden Daten aus den Wahlniederschriften gestellt. Von Anfragen an die kreisangehörigen Gemeinden oder Landkreise wurde abgesehen, da dort erfahrungsgemäß die notwendigen Verwaltungska- 
pazitäten fehlen, um derartig aufwendige Datensammlungen vorzunehmen, die im Wahlgesetz nicht vorgesehen sind.

Die in der Folge präsentierten Daten können deshalb keinen Anspruch auf Repräsentativität erheben, zumal von den angefragten Ämtern nur eine Teilmenge bereit war, die benötigten Daten aus den Wahlniederschriften zusammenzustellen. Während die Daten jedoch nicht repräsentativ sind, so decken die Fälle zumindest zahlreiche Dimensionen ab, etwa Gemeindegröße, wirtschaftliche Situation, Bundesland, Wahlebene und Wahlsystem, sodass auf dieser Basis allgemeine Tendenzen ausgemacht werden können. Insbesondere gilt bei nahezu allen untersuchten Wahlen die gleiche Schlussfolgerung: Der Anteil ungewerteter Briefwahlstimmen liegt oberhalb des offiziell berichteten Anteils ungültiger Briefwahlstimmen und der Anteil ungewerteter Briefwahlstimmen ist größer als der Anteil ungültiger Urnenwahlstimmen.

Anfragt wurden Daten für die jüngsten Kommunal- und Landtagswahlen. Einige der angefragten Gemeinden haben zusätzlich Daten für Bundestags- und Europawahlen geliefert. Diese Zahlen werden hier ebenfalls berücksichtigt, da sie einen umfassenderen Eindruck auf das Phänomen ungewerteter Briefwahlstimmen erlauben.

Der Datensatz umfasst 68 Wahlgänge aus 31 kreisfreien Städten in elf Bundesländern mit einem Schwerpunkt auf Wahlen zwischen den Jahren 2016 und 2020. Eine Übersicht der einbezogenen Wahlen bietet Tab. 1 dieses Beitrags. Der Datensatz besteht aus 32 Kommunalwahlen, 25 Landtagswahlen, sieben Bundestagswahlen und vier Europawahlen. Von den einbezogenen Wahlen fanden 18 als Parallelwahl statt, bei denen mehrere Wahlgänge zugleich durchgeführt wurden. Dies trifft vornehmlich auf die Kommunalwahlen und die Europawahl im Mai 2019 zu. Eine Teilmenge der Gemeinden hat neben der Zahl der zurückgewiesenen Wahlbriefe auch die Zurückweisungsgründe aus den Wahlniederschriften zusammengestellt, die im zweiten Abschnitt der Analyse ausgewertet werden. Dies gilt für 41 Wahlen aus 16 Gemeinden.

Für die folgende Diskussion wird zwischen drei Mengen nichtgewerteter Stimmen unterschieden, die wie folgt definiert werden. Die erste Menge sind ungültige Stimmen, wie sie in der Wahlstatistik berichtet werden. Ungültige Stimmen treten sowohl bei der Urnen- als auch bei der Briefwahl auf, da sie sich allein auf den Stimmzettel beziehen. Bei der Briefwahl treten ungültige Stimmen nur auf, wenn der Wahlbrief zuvor von den Wahlleitungen zur Wahl zugelassen wurde. Zur Berechnung von Anteilen wird die Zahl der ungültigen Stimmen in Bezug zur Zahl der Wählerinnen und Wähler gesetzt. Bei der Urnenwahl sind das alle abgegebenen Stimmzettel, egal ob gültig oder ungültig. Bei der Briefwahl sind das alle Stimmzettel aus den zur Wahl zugelassenen Wahlbriefen, egal ob gültig oder ungültig.

Die zweite Menge sind die zurückgewiesenen Stimmen. Dies umfasst die Zahl der Wahlbriefe, die von den Wahlleitungen aufgrund von Formfehlern nicht zur Wahl zugelassen wurden. Dieser Fall tritt ausschließlich bei der Briefwahl auf. Zur Berechnung von Anteilen werden die zurückgewiesenen Stimmen in Bezug zur Zahl der eingegangenen Wahlbriefe gesetzt, die der Summe aus den zugelassenen Wahlbriefen, egal ob gültig oder ungültig, und den zurückgewiesenen Wahlbriefen entspricht. 
Tab. 1 Einbezogene Wahlen

\begin{tabular}{|c|c|c|c|c|c|}
\hline Gründe & Gemeinde & Bundesland & Wahl & Wahltermin & Parallelwahl \\
\hline- & Ansbach & Bayern & Kommunal & 2020-03-15 & - \\
\hline- & Augsburg & Bayern & Landtag & 2018-10-14 & - \\
\hline- & Augsburg & Bayern & Kommunal & $2020-30-15$ & - \\
\hline- & Bonn & $\begin{array}{l}\text { Nordrhein-Westfa- } \\
\text { len }\end{array}$ & Landtag & 2017-05-14 & - \\
\hline- & Bonn & $\begin{array}{l}\text { Nordrhein-Westfa- } \\
\text { len }\end{array}$ & Kommunal & 2020-09-13 & - \\
\hline $\mathrm{a}$ & Braunschweig & Niedersachsen & Kommunal & 2016-09-11 & - \\
\hline a & Braunschweig & Niedersachsen & Landtag & $2017-10-15$ & - \\
\hline $\mathrm{a}$ & Bremen & Bremen & Landtag & 2011-05-22 & - \\
\hline a & Bremen & Bremen & Landtag & 2015-05-10 & - \\
\hline $\mathrm{a}$ & Bremen & Bremen & Bundestag & 2017-09-24 & - \\
\hline a & Bremen & Bremen & Landtag & 2019-05-26 & Europa \\
\hline $\mathrm{a}$ & Bremen & Bremen & Europa & $2019-05-26$ & Landtag \\
\hline a & Bremerhaven & Bremen & Bundestag & 2017-09-24 & - \\
\hline $\mathrm{a}$ & Cottbus & Brandenburg & Landtag & 2019-09-01 & - \\
\hline $\mathrm{a}$ & Cottbus & Brandenburg & Kommunal & 2019-05-26 & Europa \\
\hline $\mathrm{a}$ & Dessau-Roßlau & Sachsen-Anhalt & Landtag & 2016-03-13 & - \\
\hline a & Dessau-Roßlau & Sachsen-Anhalt & Bundestag & 2017-09-24 & - \\
\hline a & Dessau-Roßlau & Sachsen-Anhalt & Kommunal & 2019-05-26 & Europa \\
\hline a & Emden & Niedersachsen & Kommunal & 2016-09-11 & - \\
\hline a & Emden & Niedersachsen & Landtag & $2017-10-15$ & - \\
\hline- & Erlangen & Bayern & Kommunal & 2020-03-15 & - \\
\hline- & $\begin{array}{l}\text { Frankfurt am } \\
\text { Main }\end{array}$ & Hessen & Kommunal & 2016-03-06 & - \\
\hline- & $\begin{array}{l}\text { Frankfurt am } \\
\text { Main }\end{array}$ & Hessen & Landtag & $2018-10-28$ & - \\
\hline- & Heidelberg & Baden-Württemberg & Kommunal & 2009-06-07 & Europa \\
\hline- & Heidelberg & Baden-Württemberg & Kommunal & 2014-05-25 & Europa \\
\hline- & Heidelberg & Baden-Württemberg & Kommunal & 2019-05-26 & Europa \\
\hline a & Heilbronn & Baden-Württemberg & Landtag & 2016-03-13 & - \\
\hline- & Heilbronn & Baden-Württemberg & Kommunal & 2019-05-26 & Europa \\
\hline- & Herne & $\begin{array}{l}\text { Nordrhein-Westfa- } \\
\text { len }\end{array}$ & Kommunal & 2020-09-13 & - \\
\hline a & Jena & Thüringen & Kommunal & 2019-05-26 & Europa \\
\hline a & Jena & Thüringen & Landtag & 2019-10-27 & - \\
\hline- & Karlsruhe & Baden-Württemberg & Kommunal & 2019-05-26 & Europa \\
\hline a & Koblenz & Rheinland-Pfalz & Landtag & 2016-03-13 & - \\
\hline a & Koblenz & Rheinland-Pfalz & Kommunal & $2019-05-26$ & Europa \\
\hline- & Mannheim & Baden-Württemberg & Landtag & 2016-03-13 & - \\
\hline- & Mannheim & Baden-Württemberg & Kommunal & 2019-05-26 & Europa \\
\hline- & Nürnberg & Bayern & Landtag & 2018-10-14 & - \\
\hline- & Offenbach & Hessen & Kommunal & 2016-03-06 & - \\
\hline- & Offenbach & Hessen & Landtag & 2018-10-28 & - \\
\hline a & Oldenburg & Niedersachsen & Kommunal & 2016-09-11 & - \\
\hline
\end{tabular}


Tab. 1 (Fortsetzung)

\begin{tabular}{|c|c|c|c|c|c|}
\hline Gründe & Gemeinde & Bundesland & Wahl & Wahltermin & Parallelwahl \\
\hline a & Oldenburg & Niedersachsen & Bundestag & 2017-09-24 & - \\
\hline a & Osnabrück & Niedersachsen & Kommunal & 2016-09-11 & - \\
\hline a & Osnabrück & Niedersachsen & Bundestag & 2017-09-24 & - \\
\hline a & Osnabrück & Niedersachsen & Landtag & 2017-10-15 & - \\
\hline a & Osnabrück & Niedersachsen & Europa & $2019-05-26$ & - \\
\hline- & Rosenheim & Bayern & Kommunal & $2020-03-15$ & - \\
\hline a & Saarbrücken & Saarland & Landtag & $2017-03-26$ & - \\
\hline a & Saarbrücken & Saarland & Kommunal & 2019-05-26 & Europa \\
\hline- & Speyer & Rheinland-Pfalz & Landtag & 2016-03-13 & - \\
\hline- & Speyer & Rheinland-Pfalz & Kommunal & $2019-05-26$ & Europa \\
\hline $\mathrm{a}$ & Stuttgart & Baden-Württemberg & Landtag & 2016-03-13 & - \\
\hline a & Stuttgart & Baden-Württemberg & Kommunal & $2019-05-26$ & Europa \\
\hline a & Trier & Rheinland-Pfalz & Landtag & 2016-03-13 & - \\
\hline- & Ulm & Baden-Württemberg & Kommunal & 2009-06-07 & Europa \\
\hline- & Ulm & Baden-Württemberg & Kommunal & 2014-05-25 & Europa \\
\hline- & Ulm & Baden-Württemberg & Kommunal & $2019-05-26$ & Europa \\
\hline a & Wiesbaden & Hessen & Kommunal & 2011-03-27 & - \\
\hline a & Wiesbaden & Hessen & Bundestag & 2013-09-22 & Landtag \\
\hline a & Wiesbaden & Hessen & Landtag & 2013-09-22 & Bundestag \\
\hline a & Wiesbaden & Hessen & Europa & $2014-05-25$ & - \\
\hline $\mathrm{a}$ & Wiesbaden & Hessen & Kommunal & 2016-03-06 & - \\
\hline $\mathrm{a}$ & Wiesbaden & Hessen & Bundestag & 2017-09-24 & - \\
\hline $\mathrm{a}$ & Wiesbaden & Hessen & Landtag & 2018-10-28 & - \\
\hline $\mathrm{a}$ & Wiesbaden & Hessen & Europa & 2019-05-26 & - \\
\hline- & Wolfsburg & Niedersachsen & Kommunal & 2016-09-11 & - \\
\hline- & Wolfsburg & Niedersachsen & Landtag & $2017-10-15$ & - \\
\hline a & Wuppertal & $\begin{array}{l}\text { Nordrhein-Westfa- } \\
\text { len }\end{array}$ & Landtag & 2017-05-14 & - \\
\hline a & Wuppertal & $\begin{array}{l}\text { Nordrhein-Westfa- } \\
\text { len }\end{array}$ & Kommunal & 2020-09-13 & - \\
\hline
\end{tabular}

Bemerkung: ${ }^{\text {a }}$ Bei der Auswertung in Tab. 5 berücksichtigt

Die dritte Menge sind die ungewerteten Stimmen. Hierbei handelt es sich um die Summe aus ungültigen und zurückgewiesenen Stimmen. Auch dieser Wert ist ausschließlich relevant für die Briefwahl. Für die Berechnung von Anteilen werden die ungewerteten Stimmen in Bezug zu den im vorangegangenen Absatz definierten eingegangenen Wahlbriefen gesetzt.

Die genannten Werte werden auf Ebene der Stimmzettel ausgewiesen. Entsprechend der üblichen Konventionen in der Wahlstatistik wird der Einfachheit halber von ungewerteten Briefwahlstimmen gesprochen, wenn ungewertete Briefwahlstimmzettel gemeint sind. In den Fällen, in denen die Wahlstatistik ungültige Stimmen nur auf der Stimm- und nicht auf der Stimmzettelebene berichtet, werden die ungültigen Parteistimmen berücksichtigt, bei der Bundestagswahl also beispielsweise die Zahl der ungültigen Zweitstimmen. 
Für die Analyse liegt der Fokus auf zwei Differenzen. Einerseits wird die Differenz zwischen dem Anteil der ungewerteten Briefwahlstimmen und dem Anteil der ungültigen Briefwahlstimmen betrachtet. Je größer diese Differenz, desto stärker unterschätzt die Wahlstatistik, wie viele Briefwahlstimmen letztlich ungewertet bleiben. Der zweite Vergleich ist die Differenz zwischen dem Anteil der ungewerteten Briefwahlstimmen und dem Anteil der ungültigen Urnenwahlstimmen. Während die Wahlstatistik typischerweise eine geringere Quote ungültiger Briefwahl- als ungültiger Urnenwahlstimmen ausweist, vermittelt die hier verwendete Differenz einen besseren Eindruck davon, welcher Anteil der Briefwahlstimmen relativ zu den Urnenwahlstimmen unberücksichtigt bleibt.

Zur Einordnung der Zahlen seien einige Bemerkungen zur Briefwahl und zur Wahlstatistik vorausgeschickt. Erstens ist zu bemerken, dass Wählerinnen und Wähler mit einem Wahlschein auch am Wahltag im Wahllokal wählen können. In der Praxis wird von dieser Möglichkeit nur selten Gebrauch gemacht, sodass der Großteil der abgegebenen Wahlscheine auch tatsächlich für die Briefwahl verwendet wird. Auf die Analyse wirken sich solche Fälle nicht aus. Urnenwählerinnen und -wähler mit Wahlschein werden in den Urnenwahlbezirken ausgezählt und diese Wahlform ist auch in der Sache eher mit der Urnenwahl vergleichbar. Urnenwählerinnen und -wähler mit Wahlschein erhalten im Wahllokal einen einfachen Stimmzettel zum Einwurf in die Wahlurne, sodass die Zurückweisung des Wahlbriefs hier nicht auftreten kann.

Die Urnenwahl mit Wahlschein ist zu unterscheiden von Wählerinnen und Wählern, die ihren Wahlschein persönlich beantragen und auch direkt wählen. In diesen Fällen erhalten die Wählerinnen und Wähler die vollständigen Briefwahlunterlagen und der ausgefüllte Wahlbrief wird von den Wahlleitungen wie ein regulärer Wahlbrief bis zum Wahltag aufbewahrt und zum Wahltermin für die Auszählung an die Wahlbezirksleitungen weitergeleitet. Diese Fälle sind eher mit der Briefwahl vergleichbar, da die Wählerinnen und Wähler mit den komplexen Wahlbriefunterlagen umgehen müssen und eine Zurückweisung des Wahlbriefs durchaus denkbar ist.

Schließlich ist zu bemerken, dass die berichteten Werte ungewerteter Wahlbriefe eine konservative Schätzung des Phänomens darstellen. Von den ausgegebenen Wahlbriefunterlagen wird nur eine Teilmenge an die Wahlleitungen zurückgesendet. Während der Großteil der fehlenden Wahlbriefe sich ohne Zweifel darauf zurückführen lässt, dass die Wählerinnen und Wähler die Wahlunterlagen nicht ausfüllen und an die Wahlleitungen zurückschicken, ist nicht auszuschließen, dass einzelne Wahlbriefe auf dem Postweg verloren gehen. Während dieser Effekt prinzipiell nicht zu beobachten und aller Wahrscheinlichkeit nach eher nachrangig ist, gibt es einen weiteren Faktor, der zu einer systematischen Unterschätzung der ungewerteten Wahlbriefe führt. Die in diesem Bericht ausgewerteten Zahlen basieren auf den Niederschriften, die von den Wahlbezirksleitungen unmittelbar nach der Wahlauszählung angefertigt werden. Die Niederschriften können somit keine Zahlen über verspätet eingegangene Wahlbriefe enthalten, sodass die in der Folge dargestellten Werte die Untergrenze der ungewerteten Wahlbriefe beschreiben. 


\section{Ungewertete Wahlbriefe bei deutschen Wahlen}

In diesem Abschnitt werden die ungewerteten Briefwahlstimmen bei ausgewählten deutschen Wahlen untersucht. Im ersten Unterabschnitt wird zunächst das Gesamtaufkommen ungewerteter Briefwahlstimmen in den Blick genommen und mit den ungültigen Briefwahlwahlstimmen sowie den ungültigen Urnenwahlstimmen verglichen. Weiterhin werden die Ergebnisse von zwei Regressionsanalysen dargestellt, die das Aufkommen ungewerteter Briefwahlstimmen in Bezug zur Wahlebene und $\mathrm{zu}$ verschiedenen Strukturfaktoren setzen. Im zweiten Unterabschnitt werden die Gründe für die Zurückweisung der Briefwahlstimmen betrachtet.

\subsection{Das Aufkommen ungewerteter Wahlbriefe}

Tab. 2 stellt die deskriptiven Statistiken der verschiedenen Mengen nicht gewerteter Briefwahlstimmen bei den 68 betrachteten Wahlen dar. Die Tabelle unterstreicht klar, dass die typischerweise niedrigen Anteile ungültiger Briefwahlstimmen nur in einem engen Sinne zutreffen. Selbst bei einer konservativen Schätzung aufgrund der fehlenden Daten über verspätet eingegangene Wahlbriefe bleiben bei den 68 betrachteten Wahlen im Durchschnitt 3,6\% der Wahlbriefe unberücksichtigt, was einem um 2,4 Prozentpunkte erhöhten Wert gegenüber dem offiziell berichteten Anteil ungültiger Briefwahlstimmen entspricht. Verglichen mit dem durchschnittlichen Anteil ungültiger Urnenwahlstimmen von 1,9\% liegt der Anteil ungewerteter Briefwahlstimmen um immerhin 1,7 Prozentpunkte höher.

Um diese Befunde näher zu beleuchten, wurde das Aufkommen zurückgewiesener und ungewerteter Briefwahlstimmen in zwei Regressionsmodellen untersucht. Dabei wurden die beobachteten Anteile nicht gewerteter Briefwahlstimmen in den 68 Gemeinden durch die vier Wahlebenen erklärt. Die in den Modellen vorhergesagten Anteile zurückgewiesener und ungewerteter Briefwahlstimmen sind in Abb. 2 dargestellt. ${ }^{4}$ Die zugrundeliegenden Regressionsmodelle sind in Tab. 3 wiedergegeben.

Im Ergebnis fallen die höheren Anteile ungewerteter Wahlbriefe bei Europa- und vor allem bei Kommunalwahlen auf. An dieser Stelle kommen mehrere Effekte zusammen. Zum einen sind die Kommunalwahlsysteme in den meisten Bundesländern deutlich komplexer als die Wahlsysteme, die bei Landes-, Bundes- und Europawahlen zur Anwendung kommen, was den höheren Anteil ungültiger Brief- und Urnenwahlstimmen erklärt (Nyhuis 2014). Aber auch der Anteil zurückgewiesener Wahlbriefe liegt bei Kommunalwahlen sichtlich über den Werten, die bei Wahlen auf den höheren politischen Ebenen zu beobachten sind.

Zur Erklärung dieser Unterschiede liegen zumindest drei Effekte auf der Hand. Einerseits besitzen bei Kommunal- und Europawahlen mehr Einwohnerinnen und Einwohner das Wahlrecht als bei Bundestags- und Landtagswahlen. Eine unterschiedliche Zusammensetzung der Wählerschaft ist eine plausible Erklärung für die

\footnotetext{
4 Wie in der empirischen Forschungsliteratur zunehmend üblich, wurden sowohl das $80 \%$ - als auch das $95 \%$-Konfidenzintervall angegeben, um die Unsicherheit der Schätzung für unterschiedliche Irrtumswahrscheinlichkeiten darzustellen.
} 
Tab. 2 Deskriptive Statistiken

\begin{tabular}{lllll}
\hline & $\begin{array}{l}\text { Durchschnittlicher } \\
\text { Anteil }\end{array}$ & $\begin{array}{l}\text { Standard- } \\
\text { abweichung }\end{array}$ & Minimum & Maximum \\
\hline (1) Ungültige Briefwahl & 1,2 & 0,9 & 0,1 & 4,9 \\
$\begin{array}{l}\text { (2) Zurückgewiesene Wahl- } \\
\text { briefe }\end{array}$ & 2,5 & 1,3 & 0,2 & 7,6 \\
(3) Ungewertete Briefwahl & 3,6 & 1,7 & 0,4 & 11,2 \\
Differenz (3)-(1) & 2,4 & 1,2 & 0,1 & 6,3 \\
(4) Ungültige Urnenwahl & 1,9 & 1,1 & 0,5 & 5,9 \\
Differenz (3)-(4) & 1,7 & 1,2 & $-0,4$ & 5,6 \\
\hline
\end{tabular}

Bemerkung: Die Werte beziehen sich auf 68 Wahlgänge in 31 kreisfreien Städten zwischen 2009 und 2020. Die durchschnittlichen Anteile in Zeile (3) ergeben sich nicht als Summe der Zeilen (1) und (2), da die Prozentuierungsbasis für Zeile (1) die Zahl der zur Wahl zugelassenen Wahlbriefe, in den Zeilen (2) und (3) dagegen die eingegangenen Wahlbriefe sind

Tab. 3 Wahlspezifische Unterschiede (Lineare Einfachregressionen)

\begin{tabular}{lllll}
\hline & \multicolumn{2}{l}{ Anteil zurückgewiesener Wahlbriefe } & \multicolumn{2}{l}{ Anteil ungewerteter Briefwahlstimmen } \\
& Modell 1 & Modell 2 & Modell 3 & Modell 4 \\
\hline Bundestagswahl & - & $-0,990$ & - & $-1,849^{* *}$ \\
& - & $(0,519)$ & - & $(0,671)$ \\
Landtagswahl & - & $-0,931^{* *}$ & - & $-1,446^{* *}$ \\
& - & $(0,332)$ & - & $(0,429)$ \\
Europawahl & - & $-0,244$ & - & $-1,306$ \\
& - & $(0,659)$ & - & $(0,852)$ \\
Konstante & $2,460^{* * *}$ & $2,919^{* * *}$ & $3,601^{* * *}$ & $4,406^{* * *}$ \\
& $(0,158)$ & $(0,220)$ & $(0,212)$ & $(0,284)$ \\
$N$ & 68 & 68 & 68 & 68 \\
$\mathrm{R} 2$ & - & 0,128 & - & 0,194 \\
\hline
\end{tabular}

Bemerkung: $* * * p<0,001$; $* * p<0,01 ; * p<0,05$; Referenzkategorie: Kommunalwahl; Standardfehler in Klammern

höheren Zurückweisungsraten bei der Briefwahl. Gerade wenn sich EU-Bürgerinnen und -Bürger mit einem Wahlbrief an den Kommunalwahlen beteiligen, dann könnten etwa Sprachbarrieren zu einem höheren Anteil zurückgewiesener Wahlbriefe führen. Daneben hat auch das Wahlrecht für 16- und 17-Jährige einen immer größeren Stellenwert und wird besonders auf der kommunalen Ebene mittlerweile in vielen Bundesländern praktiziert (Leininger und Faas 2020). Auch durch diesen Faktor könnten sich erhöhte Anteile zurückgewiesener Wahlbriefe erklären, wenn sich mehr unerfahrene Wählerinnen und Wähler an kommunalen Wahlgängen beteiligen. Andererseits ist die Mehrzahl der hier untersuchten Kommunalwahlen parallel mit der Europawahl durchgeführt worden. Das ohnehin schon komplexe Briefwahlverfahren wird durch eine Parallelwahl noch komplexer, da weitere Briefwahlunterlagen von den Wählerinnen und Wählern gehandhabt werden müssen, was die Fehlerpotenziale erhöht.

Um den Effekt dieser drei Faktoren auf den Anteil zurückgewiesener Wahlbriefe zu untersuchen, wurden zwei weitere Regressionsmodelle berechnet, die den Anteil 
Abb. 2 Wahlspezifische Unterschiede der a zurückgewiesenen und $\mathbf{b}$ ungewerteten Briefwahlstimmen. Bemerkung: Die Vorhersagen basieren auf zwei linearen Einfachregressionen. Die Modelle sind in Tab. 3 dargestellt. Die hervorgehobenen Striche geben das $80 \%$-Konfidenzintervall an, das $95 \%$ Konfidenzintervall ist durch die dünnen Linien angegeben. Die vorhergesagten Punktwerte sind in Klammern angegeben. Die unterschiedlich großen Konfidenzintervalle ergeben sich durch die unterschiedlichen Fallzahlen für die verschiedenen Wahlgänge

\section{a}

Kommunalwahl

Europawahl

Landtagswahl

Bundestagswahl

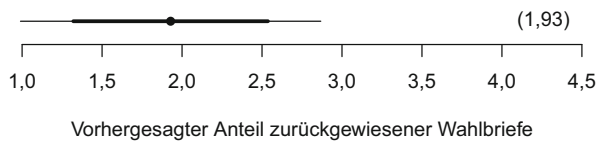

b

Kommunalwahl

Europawahl

$(3,10)$

Landtagswahl

Bundestagswahl

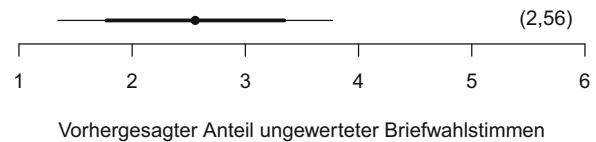

zurückgewiesener und ungewerteter Wahlbriefe mit dem Wahlrecht für EU-Ausländerinnen und -Ausländer, dem Jugendwahlrecht und der Parallelwahl erklären. Das Ergebnis dieser Analyse ist in Tab. 4 dargestellt.

Die Modelle legen nahe, dass besonders das Wahlrecht für EU-Ausländerinnen und -Ausländer zu erhöhten Anteilen zurückgewiesener Wahlbriefe führt. Auch die Koeffizienten für die Parallelwahl weisen in eine positive Richtung, allerdings sind 
Tab. 4 Determinanten ungewerteter Briefwahlstimmen (Lineare Einfachregressionen)

\begin{tabular}{lll}
\hline & $\begin{array}{l}\text { Anteil zurückgewie- } \\
\text { sener Wahlbriefe }\end{array}$ & $\begin{array}{l}\text { Anteil ungewerteter } \\
\text { Briefwahlstimmen }\end{array}$ \\
\hline Wahlrecht & $0,814^{*}$ & $1,396^{* *}$ \\
Europäer & $(0,367)$ & $(0,484)$ \\
Wahlrecht & $-0,050$ & $-0,157$ \\
16/17-Jährige & $(0,361)$ & $(0,477)$ \\
Parallelwahl & 0,280 & 0,138 \\
& $(0,403)$ & $(0,533)$ \\
Konstante & $1,972^{* * *}$ & $2,887 * * *$ \\
& $(0,224)$ & $(0,295)$ \\
$N$ & 68 & 68 \\
R2 & 0,132 & 0,162 \\
\hline
\end{tabular}

Bemerkung: $* * * p<0,001 ; * * p<0,01 ; * p<0,05$; Standardfehler in Klammern

diese Werte nicht signifikant von null verschieden. Ein Effekt des Jugendwahlrechts kann ebenfalls nicht beobachtet werden. Der Befund, dass besonders das Ausländerwahlrecht zu erhöhten Anteilen von Formfehlern bei der Briefwahl führt, mag einerseits auf die fehlende Vertrautheit mit dem deutschen Wahlsystem zurückgeführt werden, andererseits kann dieser Effekt aber auch in Sprachbarrieren begründet liegen. Die Möglichkeit von Sprachbarrieren liegt schon deshalb nahe, da die deutschen Briefwahlunterlagen ausgesprochen textlastig sind und entsprechende Verständnisschwierigkeiten hervorrufen können. Neben der Reform der Briefwahlunterlagen durch Kürzung der Textelemente, ergibt sich aus diesem Befund deshalb noch eine zweite mögliche Reformoption, nämlich die international durchaus praktizierte Übersetzung von Wahlmaterialien (Hopkins 2011; Jones-Correa 2005).

Unabhängig von den Determinanten ungewerteter Briefwahlstimmen, weisen die dargestellten Befunde aber auch auf grundsätzliche Schwierigkeiten mit der Briefwahl hin, die in aktuellen Diskussionen um die Briefwahl nicht ausreichend berücksichtigt werden. Ungesehen gehen bei der Briefwahl deutlich mehr Stimmen verloren als bei der Urnenwahl. Um zu untersuchen, an welcher Stelle die Wählerinnen und Wähler am Wahlbriefverfahren scheitern, wendet sich der folgende Abschnitt den Gründen für die Zurückweisung von Wahlbriefen zu.

\subsection{Gründe für die Zurückweisung der Briefwahl}

Die Niederschriften aus den Wahlbezirken erfassen nicht nur die Menge der zurückgewiesenen Wahlbriefe, auch die Zurückweisungsgründe werden zum Zwecke einer möglichen Wahlprüfung festgehalten. Dazu weisen die Vordrucke der Wahlniederschriften Felder für sieben der acht Zurückweisungsgründe aus. Der verspätete Eingang der Wahlbriefe (Grund 1) wird durch die Wahlbezirksleitungen nicht erfasst, da die Niederschriften unmittelbar nach der Wahlauszählung erstellt werden und verspätetet eingegangene Wahlbriefe zu diesem Zeitpunkt nicht vorliegen.

Für 41 Wahlen aus 16 Gemeinden konnten Daten über das Aufkommen der verschiedenen Zurückweisungsgründe gewonnen werden. Die deskriptiven Statistiken 
Tab. 5 Gründe für die Zurückweisung von Wahlbriefen (in Prozent)

\begin{tabular}{|c|c|c|c|c|}
\hline Grund & Durchschnitt & $\begin{array}{l}\text { Standard- } \\
\text { abweichung }\end{array}$ & Minimum & Maximum \\
\hline Fehlender Wahlschein (Grund 2) & 68,0 & 10,9 & 43,4 & 89,3 \\
\hline $\begin{array}{l}\text { Kein Stimmzettelumschlag } \\
\text { (Grund 3) }\end{array}$ & 6,3 & 5,2 & 0,9 & 23,2 \\
\hline Briefe nicht verschlossen (Grund 4) & 4,4 & 3,9 & 0,0 & 14,0 \\
\hline $\begin{array}{l}\text { Nicht ausreichend Wahlscheine } \\
\text { (Grund 5) }\end{array}$ & 0,8 & 1,0 & 0,0 & 4,5 \\
\hline Fehlende Unterschrift (Grund 6) & 13,2 & 9,0 & 0,7 & 39,9 \\
\hline Kein amtlicher Umschlag (Grund 7) & 4,1 & 4,6 & 0,0 & 22,8 \\
\hline Wahlgeheimnis verletzt (Grund 8) & 3,2 & 4,0 & 0,0 & 17,3 \\
\hline
\end{tabular}

Bemerkung: Die Werte beziehen sich auf 41 Wahlgänge in 16 kreisfreien Städten zwischen 2011 und 2020

sind in Tab. 5 dargestellt. Die Werte wurden relativ zum Gesamtaufkommen der zurückgewiesenen Wahlbriefe prozentuiert. Da der verspätete Eingang von Wahlbriefen nicht berücksichtigt werden kann, wurden die Anteile relativ zur Häufigkeit der sieben weiteren Zurückweisungsgründe berechnet.

Der mit Abstand häufigste Grund für die Zurückweisung von Wahlbriefen ist ein fehlender Wahlschein, der in mehr als zwei Dritteln der betrachteten Fälle zur Zurückweisung führt. Aber auch die fehlende Unterschrift unter der eidesstattlichen Erklärung führt in immerhin rund $13 \%$ der Fälle zur Zurückweisung des Wahlbriefs. Zusammen mit einer nicht ausreichenden Zahl von Wahlscheinen (Grund 5), sind Probleme mit dem Wahlschein also in etwa vier von fünf Fällen für die Zurückweisung des Wahlbriefs verantwortlich. Im Gegensatz dazu sind Bedenken um das Wahlgeheimnis (Gründe 4, 7 und 8) lediglich in rund jedem zehnten Fall der Zurückweisungsgrund.

Zusammenfassend kann somit festgestellt werden, dass Wählerinnen und Wähler vor allem an den zur Verhinderung von Wahlbetrug eingeführten Regelungen scheitern. Gerade diese Regelungen machen die Briefwahl komplexer und also fehleranfälliger als die Urnenwahl, was zu einem erhöhten Anteil ungewerteter Briefwahlstimmen führt.

\section{Schlussfolgerungen: Reform der Briefwahl in Deutschland}

Seit Jahren steigt der Briefwähleranteil in Deutschland. Während Wählerinnen und Wähler die Bequemlichkeit der Stimmabgabe per Wahlbrief schätzen, ist das Ziel der vorliegenden Arbeit, auf bisher unbeachtete Probleme dieser Entwicklung aufmerksam zu machen. Vor dem Hintergrund des Zusammenhangs zwischen der Komplexität des Wahlakts und der Wahrscheinlichkeit von Wählerirrtum (Herrnson et al. 2012; Kimball und Kropf 2005; Pachón et al. 2017) wurde argumentiert, dass das Briefwahlverfahren anspruchsvoller und somit fehleranfälliger als die klassische Urnenwahl ist. Es liegt deshalb nahe, dass mehr Wählerstimmen bei der Briefwahl als bei der Urnenwahl verloren gehen. In der deutschen Wahlgesetzgebung werden Wahlbriefe mit Formfehlern als nicht abgegeben gewertet, sodass sie in der 
Wahlstatistik nicht auftauchen. Aus diesem Grund werden bestehende Probleme der Briefwahl in der öffentlichen Diskussion nicht nur nicht gesehen - sie können gar nicht gesehen werden.

Die Befunde dieser Arbeit lassen sich in zwei Punkten zusammenfassen. Erstens liegen die Anteile der ungewerteten Briefwahlstimmen in den untersuchten Gemeinden im Schnitt rund 2,4 Prozentpunkte über dem offiziellen Anteil ungültiger Briefwahlstimmen und immerhin 1,7 Prozentpunkte über dem Anteil ungültiger Urnenwahlstimmen. Dabei lassen sich besonders hohe Anteile ungewerteter Briefwahlstimmen bei Europa- und Kommunalwahlen feststellen, die in Teilen mit dem Ausländerwahlrecht bei diesen Wahlgängen erklärt wurden. Zweitens wird der Großteil der Wahlbriefe aufgrund von Problemen mit dem Wahlschein zurückgewiesen.

Zur Untersuchung ungewerteter Briefwahlstimmen wurden in dieser Arbeit Daten zur Zurückweisung von Wahlbriefen bei Wahlen in ausgewählten kreisfreien Städten analysiert. Zwei Einschränkungen der gewählten Datenbasis seien an dieser Stelle noch einmal explizit genannt. Da die zurückgewiesenen Wahlbriefe von den Wahlleitungen nicht zentral gezählt und veröffentlicht werden, ist das Phänomen naturgemäß nur schwer zu erfassen. Für die vorliegende Arbeit konnte mit Unterstützung der kommunalen Wahl- und Statistikämter ein erster Eindruck des Aufkommens ungewerteter Briefwahlstimmen gewonnen werden. Dennoch können die Daten keinen Anspruch auf Repräsentativität erheben; es können lediglich allgemeine Tendenzen ausgemacht werden. Trotz dieser Einschränkung lassen sich die Erwartungen über die Beziehung zwischen ungewerteten Briefwahlstimmen, ungültigen Briefwahlstimmen und ungültigen Urnenwahlstimmen in nahezu allen untersuchten Gemeinden nachweisen. Die Vermutung liegt deshalb nahe, dass trotz der unrepräsentativen Datenbasis ein allgemeines Phänomen der deutschen Briefwahl beschrieben wurde. Eine weitere Einschränkung der Datenbasis besteht aufgrund der Unterschätzung der Menge zurückgewiesener Wahlbriefe. Da verspätet eingegangene Wahlbriefe in den Daten nicht berücksichtigt werden, wird das untersuchte Phänomen systematisch unterschätzt.

Zwei Forderungen ergeben sich aus der Untersuchung zur Reform der Briefwahl. Erstens darf die Briefwahl insgesamt als komplex und aus Sicht der Wählerinnen und Wähler als anfällig für Formfehler bezeichnet werden. Es ist nicht offensichtlich, wie das grundsätzliche Briefwahlverfahren vereinfacht werden kann und zugleich die in Abschn. 3 skizzierten Bedenken um Wahlbetrug und das Wahlgeheimnis zu berücksichtigen. Das heißt jedoch nicht, dass die deutschen Wahlbriefunterlagen nicht durchaus reformbedürftig sind - und dass eine Komplexitätsreduktion auch ohne die Änderung grundlegender Prinzipien denkbar ist. Häufig entsprechen die Briefwahldokumente weder mit Blick auf das Layout noch mit Blick auf die großen Textmengen den Standards gut gestalteter Wahlunterlagen und insbesondere nicht den Vorgaben leichter Sprache (Nyhuis et al. 2021; Bredel und Maaß 2016). Besonders notwendig scheint es, unwesentliche Informationen auf den Wahlbriefunterlagen zu kürzen und entscheidende Hinweise sowohl grafisch als auch textuell stärker hervorzuheben, damit Wählerinnen und Wähler möglichst keine unbewussten Formfehler bei der Briefwahl begehen.

Der spezifische Befund, dass besonders das Ausländerwahlrecht zu erhöhten Anteilen ungewerteter Briefwahlstimmen führt, mag darüber hinaus Anlass geben, um 
über die Übersetzung der deutschen Briefwahlunterlagen nachzudenken, wie sie in anderen Ländern bereits praktiziert wird (Hopkins 2011; Jones-Correa 2005). Gerade wenn Sprachbarrieren erhöhte Zurückweisungszahlen erklären, dann könnten übersetzte Wahlunterlagen derartige Fehler geringer ausfallen lassen.

Damit einher geht die Forderung nach bundesweit einheitlichen Standards für die Ausgestaltung von Briefwahlunterlagen. Hier gibt es mitunter erhebliche Varianz zwischen den Ländern und manche Dokumente werden den Anforderungen gelungener Gestaltung besser gerecht als andere. In der Erwartung, dass schlecht gestaltete Briefwahlunterlagen den Anteil ungewerteter Briefwahlstimmen erhöhen, gibt es gute Gründe für die Formulierung von empirisch abgesicherten und bundesweit einheitlichen Best-Practice-Regelungen.

Zweitens sollten Zahlen über die zurückgewiesenen Wahlbriefe künftig in der Wahlstatistik ausgewiesen werden. Konkret sollte die Zahl der zurückgewiesenen Wahlbriefe in den einzelnen Wahlbezirken ausgewiesen werden und im Idealfall auch die Menge der verschiedenen Zurückweisungsgründe. Unabhängig davon, ob einzelne Gründe ausgewiesen werden, sollten die verspätet eingegangenen Wahlbriefe zu der Zahl der zurückgewiesenen Wahlbriefe hinzugefügt werden. Hier könnte ein Ausschlussdatum die Finalität und Vergleichbarkeit der Wahlstatistik garantieren, sodass verspätete Wahlbriefe beispielsweise bis zu drei Monate nach dem Wahldatum in der Wahlstatistik berücksichtigt werden. Der Mehraufwand für die Wahlleitungen durch die genannten Änderungen ist überschaubar. Wie bemerkt liegen die erforderlichen Daten - mit Ausnahme der verspätet eingegangenen Wahlbriefe - ohnehin in den Niederschriften der Wahlbezirke vor, sodass die Daten lediglich zentral gesammelt und veröffentlicht werden müssten.

Einerseits könnte die empirische Forschung zur deutschen Briefwahl mithilfe solcher Daten auf ein solideres Fundament gestellt werden. Die Forschungspotenziale liegen auf der Hand. Nicht nur könnten auf diese Weise problematische Zusammenhänge im Aufkommen zurückgewiesener Wahlbriefe systematisch nachvollzogen werden - werden beispielsweise in bestimmten Wahlbezirken besonders viele Wahlbriefe zurückgewiesen? -, auch mögliche Hinweise zur Reform des Wahlbriefverfahrens könnten durch die Zahl und Gründe der zurückgewiesenen Wahlbriefe empirisch fundiert werden.

Schließlich ist es aber vornehmlich ein Gebot der Transparenz des Wahlakts, dass die Menge der zurückgewiesenen Wahlbriefe veröffentlicht wird. Nicht nur können so denkbare Unregelmäßigkeiten identifiziert werden. Vor allem erhalten Wählerinnen und Wähler die Gelegenheit, sich eine Meinung über das Risiko einer ungewerteten Briefwahlstimme zu bilden.

\footnotetext{
Danksagung Ein besonderer Dank gilt den Mitarbeiterinnen und Mitarbeitern der kommunalen Wahlund Statistikämter, die sich um die Zusammenstellung der hier ausgewerteten Daten bemüht haben, insbesondere Evelyn Temme vom Landeswahlamt Bremen, die mich auf die Problematik der ungewerteten Briefwahlstimmen aufmerksam gemacht hat. Ein weiterer Dank gilt den Gutachter/innen sowie dem Herausgeber/innenteam der Politischen Vierteljahresschrift.
}

Conflict of Interest D. Nyhuis has no competing interests to declare that are relevant to the content of this article. 


\section{Literatur}

Alvarez, R. Michael, Dustin Beckett, und Charles Stewart III. 2013. Voting technology, vote-by-mail, and residual votes in California, 1990-2010. Political Research Quarterly 66(3):658-670.

Alvarez, R. Michael, Thad E. Hall, und Betsy Sinclair. 2008. Whose absentee votes are returned and counted: the variety and use of absentee ballots in california. Electoral Studies 27(4):673-683.

Ansolabehere, Stephen, und Charles Stewart III. 2005. Residual votes attributable to technology. Journal of Politics 67(2):365-389.

Baasen, Geert. 2005. Der unbekannte Ungültigwähler: Protest oder Unvermögen? Ergebnisse der Untersuchung ungültiger Stimmzettel bei der Europawahl 2004 in Berlin. Berlin: Berliner Statistik.

Barreto, Matt A., Matthew J. Streb, Mara Marks, und Fernando Guerra. 2006. Do absentee voters differ from polling place voters? New evidence from California. Public Opinion Quarterly 70(2):224-234.

Berinsky, Adam J., Nancy Burns, und Michael W. Traugott. 2001. Who votes by mail? A dynamic model of the individual-level consequences of voting-by-mail systems. Public Opinion Quarterly 65(2):178-197.

Bredel, Ursula, und Christiane Maaß. 2016. Leichte Sprache: Theoretische Grundlagen, Orientierung für die Praxis. Berlin: Dudenverlag.

Buchler, Justin, Matthew Jarvis, und John E. McNulty. 2004. Punch card technology and the racial gap in residual votes. Perspectives on Politics 2(3):517-524.

Buchstein, Hubertus. 2000. Präsenzwahl, Briefwahl, Onlinewahl und der Grundsatz der geheimen Stimmabgabe. Zeitschrift für Parlamentsfragen 31(4):886-902.

Bundeswahlleiter. 2018. Wahl zum 19. Deutschen Bundestag am 24. September 2017. Heft 5, Teil 2, Textliche Auswertung (Repräsentative Wahlstatistik und Wahlbezirksstatistik). Wiesbaden: Bundeswahlleiter.

Carman, Christopher, James Mitchell, und Robert Johns. 2008. The unfortunate natural experiment in ballot design: the Scottish parliamentary elections of 2007. Electoral Studies 27(3):442-459.

Driscoll, Amanda, und Michael J. Nelson. 2014. Ignorance or opposition? Blank and spoiled votes in lowinformation, highly politicized environments. Political Research Quarterly 67(3):547-561.

Fatke, Matthias, und Till Heinsohn. 2017. Invalid voting in German constituencies. German Politics 26(2):273-291.

Gerber, Alan S., Gregory A. Huber, und Seth J. Hill. 2013. Identifying the effect of all-mail elections on turnout: staggered reform in the evergreen state. Political Science Research and Methods 1(1):91-116.

Gronke, Paul, und Peter Miller. 2012. Voting by mail and turnout in oregon: revisiting Southwell and Burchett. American Politics Research 40(6):976-997.

Gronke, Paul, Eva Galanes-Rosenbaum, Peter A. Miller, und Daniel Toffey. 2008. Convenience voting. Annual Review of Political Science 11:437-455.

Hanmer, Michael J., und Michael W. Traugott. 2004. The impact of voting by mail on voter behavior. American Politics Research 32(4):375-405.

Hanmer, Michael J., Won-Ho Park, Michael W. Traugott, Richard G. Niemi, Paul S. Herrnson, Benjamin B. Bederson, und Frederick G. Conrad. 2010. Losing fewer votes: the impact of changing voting systems on residual votes. Political Research Quarterly 63(1):129-142.

Herrnson, Paul S., Michael J. Hanmer, und Richard G. Niemi. 2012. The impact of ballot type on voter errors. American Journal of Political Science 56(3):716-730.

Herron, M.C., und J.S. Sekhon. 2003. Overvoting and representation: an examination of overvoted presidential ballots in Broward and Miami-Dade counties. Electoral Studies 22(1):21-47.

Hopkins, Daniel J. 2011. Translating into votes: the electoral impacts of Spanish-language ballots. American Journal of Political Science 55(4):813-829.

Jones-Correa, Michael. 2005. Language provisions under the voting rights act: How effective are they? Social Science Quarterly 86(3):549-564.

Karp, Jeffrey A., und Susan Banducci. 2000. Going postal: how all-mail elections influence turnout. Political Behavior 22:223-239.

Karp, Jeffrey A., und Susan A. Banducci. 2001. Absentee voting, mobilization, and participation. American Politics Research 29(2):183-195.

Kersting, Norbert. 2019. Wahlgeheimnis vor dem Aus? Globaler Normenwandel. Zeitschrift für Parteienwissenschaften 25(2):212-219.

Kimball, David C., und Martha Kropf. 2005. Ballot design and unrecorded votes on paper-based ballots. Public Opinion Quarterly 69(4):508-529.

Kimball, David C., und Martha Kropf. 2008. Voting technology, ballot measures, and residual votes. American Politics Research 36(4):479-509. 
Knack, Stephen, und Martha Kropf. 2003. Voided ballots in the 1996 Presidential election: a county-level analysis. Journal of Politics 65(3):881-897.

Kouba, Karel, und Jakub Lysek. 2019. What affects invalid voting? A review and meta-analysis. Government and Opposition 54(4):745-775.

Kousser, Thad, und Megan Mullin. 2007. Does voting by mail increase participation? Using matching to analyze a natural experiment. Political Analysis 15(4):428-445.

Lavies, Ralf-Rainer. 1968. Die ungültige Stimmabgabe: Absicht oder Absichtslosigkeit. Politische Vierteljahresschrift 9(2):212-222.

Leininger, Arndt, und Thorsten Faas. 2020. Votes at 16 in Germany: examining subnational variation. In Lowering the voting age to 16: learning from real experiences worldwide, Hrsg. Jan Eichhorn, Johannes Bergh, 143-166. Cham: Palgrave Macmillan.

McAllister, Ian, und Toni Makkai. 1993. Institutions, society or protest? Explaining invalid votes in Australian elections. Electoral Studies 12(1):23-40.

Moral, Mert. 2016. The passive-agressive voter: the calculus of casting an invalid vote in European democracies. Political Research Quarterly 69(4):732-745.

Nyhuis, Dominic. 2014. Mehr Stimmen gleich bessere demokratische Qualität? Negative Effekte der Komplexität des Wahlrechts auf die Zusammensetzung der Wählerschaft. Zeitschrift für Politikwissenschaft 24(3):255-280.

Nyhuis, Dominic, Michael Jankowski, Felix Münchow, Morten Harmening, Jannik Bruns, Max Holly, Josip Karacic, und Christoph Hönnige. 2021. Stimmzettel nutzerfreundlicher gestalten: Empfehlungen auf Grundlage eines Survey-Experiments. Politische Vierteljahresschrift 62:93-119.

Orlowski, Frederik, und Simon Pohlmann. 2020. Die Briefwahl: Ein scharfes Schwert im Kampf gegen Epidemien? Überlegungen anlässlich kommunaler „Zwangsbriefwahlen“. Zeitschrift für Parteienwissenschaften 26(1):38-43.

Pachón, Mónica, Royce Carroll, und Hernando Barragán. 2017. Ballot design and invalid votes: Evidence from Colombia. Electoral Studies 48:98-110.

Pollex, Jan, Sebastian Block, Martin Gross, Dominic Nyhuis, und Jan A. Velimsky. 2021. Ein zunehmend bunter Freistaat: Die Analyse der bayerischen Kommunalwahlen im März 2020 unter besonderer Berücksichtigung der kreisfreien Städte. Zeitschrift für Parlamentsfragen 52(1):78-94.

Power, Timothy J., und James C. Garand. 2007. Determinants of invalid voting in Latin America. Electoral Studies 26(2):432-444.

Richey, Sean. 2008. Voting by mail: turnout and institutional reform in Oregon. Social Science Quarterly 89(4):902-915.

Sinclair, D.E., und R. Michael Alvarez. 2004. Who overvotes, who undervotes, using punchcards? Evidence from Los Angeles county. Political Research Quarterly 57(1):15-25.

Solvak, Mihkel, und Kristjan Vassil. 2015. Indifference or indignation? Explaining purposive vote spoilling in elections. Journal of Elections, Public Opinion and Parties 25(4):463-481.

Southwell, Priscilla L. 2004. Five years later: a re-assessment of Oregon's vote by mail electoral process. Political Science and Politics 37(1):89-93.

Southwell, Priscilla L. 2009. Analysis of the turnout effects of vote by mail elections, 1980-2007. Social Science Journal 46(1):211-216.

Southwell, Priscilla L., und Justin Burchett. 2000. Does changing the rules change the players? The effect of all-mail elections on the composition of the electorate. Social Science Quarterly 81(3):837-845.

Southwell, Priscilla L., und Justin I. Burchett. 2000. The effect of all-mail elections on voter turnout. American Politics Research 28(1):72-79.

Thompson, Daniel M., A. Wu Jennifer, Jesse Yoder, und Andrew B. Hall. 2020. Universal vote-by-mail has no impact on partisan turnout or vote share. Proceedings of the National Academy of Sciences 117(25):14052-14056.

Tomz, Michael, und Robert P. van Houweling. 2003. How does voting equipment affect the racial gap in voided ballots? American Journal of Political Science 47(1):46-60.

Uggla, Fredrik. 2008. Incompetence, alienation, or calculation? Explaining levels of invalid ballots and extra-parliamentary votes. Comparative Political Studies 41(8):1141-1164.

Vetter, Angelika. 2015. Just a matter of timing? Local electoral turnout in Germany in the context of national and European parliamentary elections. German Politics 24(1):67-84.

Wagner, Aiko, und Josephine Lichteblau. Im Erscheinen. Germany going postal? Comparing postal and election day voters in the 2017 German federal election. German Politics. https://doi.org/10.1080/ 09644008.2020.1759553. 
Wand, Jonthan N., Kenneth W. Shotts, Jasjeet S. Sekhon, Walter R. Mebane Jr., Michael C. Herron, und Henry E. Brady. 2001. The butterfly did it: the abberant vote for Buchanan in palm beach county, Florida. American Political Science Review 95(4):793-810.

Warf, Barney. 2006. Voting technologies and residual ballots in the 2000 and 2004 presidential elections. Political Geography 25(5):530-556.

Zucco, Cesar, Jr., und Jairo M. Nicolau. 2016. Trading old errors for new errors? The impact of electronic voting technology on party label votes in Brazil. Electoral Studies 43:10-20. 